\title{
The Influence of Experimental Pain on Primary Motor Cortex Function: Protocol for a Systematic Review and Meta-analysis
}

Nahian Chowdhury ( $\nabla$ n.chowdhury@neura.edu.au )

Neuroscience Research Australia

Wei-Ju Chang

Neuroscience Research Australia

Samantha K Millard

Neuroscience Research Australia

Patrick Skippen

Neuroscience Research Australia

Katarzyna Bilska

Neuroscience Research Australia

David S Seminowicz

University of Maryland School of Dentistry

Siobhan M Schabrun

Neuroscience Research Australia

Protocol

Keywords: Experimental Pain, Primary motor cortex, Transcranial Magnetic Stimulation

Posted Date: June 4th, 2020

DOI: https://doi.org/10.21203/rs.3.rs-33009/v1

License: (9) (i) This work is licensed under a Creative Commons Attribution 4.0 International License. Read Full License 


\section{Abstract}

Introduction: The primary motor cortex (M1) is a key brain region implicated in pain processing. Here, we present a protocol for a review that aims to synthesise and critically appraise the evidence for the effect of experimentalpain on M1 function.

Methods/Analysis: A systematic review and meta-analysis will be conducted. Electronic databases will be searched using a predetermined strategy. Studies published before April 2020 that investigate the effects of experimentally induced pain on corticomotor excitability (CME) in healthy individuals will be included if they meet eligibility criteria. Study identification, data extraction andrisk of bias assessment will be conducted by two independent reviewers, with a third reviewer consulted for any disagreements. The primary outcomes will include group level changes in CME and intracortical, transcortical and sensorimotor modulators of CME. A separate analysis using individual data will also be conducted to explore individual differences in $\mathrm{CME}$ in response to experimental pain. The meta-analysis will consider the following factors: pain model (transient, tonic, transitional pain), type of painful tissue (cutaneous, musculoskeletal), time points of outcome measures(during or after recovery from pain) and localisation of pain(target area, control area).

Discussion: This review will provide a comprehensive understanding of the mechanisms within M1 that mediate experimentally induced pain, both on a group and individual level.

Registration Number: The systematic review is registered with the International Prospective Register of Systematic Reviews (\#CRD42020173172)

\section{Introduction}

Musculoskeletal conditions associated with pain are a leading cause of disability worldwide[1]. Despite considerable research effort, effective treatments remain elusive. This can in part, be attributed to a limited understanding of the neurophysiological mechanisms that mediate pain. To understand these mechanisms, studies have used experimental models to induce transient pain (momentary pain which resolves within seconds)[2],tonic pain (pain that is sustained over several minutes) [3]or transitional pain (i.e. delayed onset pain sustained over several days or weeks)[4]in healthy individuals while concurrently investigating the effects of pain on brain activity. One brain region of interest in the study of pain is the primary motor cortex (M1).

M1 function has typically been studied by delivering single pulses of transcranial magnetic stimulation (TMS) to M1 and measuring the associated motor evoked potentials (MEPs) from a peripheral muscle, which is used toindex of corticomotor excitability (CME) $[5,6]$. Besides single pulse TMS, other TMS paradigms have been utilised to assess alterations in intracortical (within M1), transcortical (between left and right M1s) or sensorimotor (between sensory input and M1 processing) circuits that inhibit or facilitate CME[7-9]. On a group level, these studies reveal significant changes in CME as well as intracortical, transcortical and sensorimotor circuits in response to experimentalpain. For example, 
studies have shown a reduction in CME during and after recovery from tonicpain relative to baseline[8, 10], as well as an increase in intracortical inhibition after recovery from tonic pain[10]. However, recent studies have also begun to consider individual differences in CME in response to experimentalpain[1113]. One study [13]showed two distinct responses to transitionalpain: facilitation of CME and suppression of CME. Those who showed facilitation of CME relative to a pain-free baseline exhibited less pain than those who showed suppression of CME. This finding suggests that understanding individual differences may be of critical importance in explaining why some people develop more severe pain than others.

Here we present a protocol for a systematic review and meta-analyses that aims to qualitatively and quantitatively synthesise the available literature and critically appraise the evidence foraltered M1 functionin response to experimentally induced pain, both at the group and individual level. One previous systematic review[14] has assessed the effect of experimentally induced tonic musculoskeletal pain on $\mathrm{CME}$, and two other systematic reviews have investigated TMS measures in clinical pain $[15,16]$. However, there has yet to be a synthesis of literature investigating the effect of transient or transitional pain on $\mathrm{CME}$, the effect of pain on modulators of $\mathrm{CME}$, and how individual differences in CME are associated with pain severity. Such a synthesis will provide a bigger picture of alterations in M1 function in response to experimental pain.

\section{Methods/designs}

This protocol is prepared in accordance with the Preferred Reporting Items for Systematic

Review and Meta-analysis Protocols (PRISMA-P) guidelines[17]. The review was prospectively registered with the International Prospective Register of Systematic Reviews (PROSPERO; registration number: CRD42020173172).

\section{Review Questions}

Question 1. Is corticomotor excitability, and/or intracortical, transcortical and sensorimotor modulators of $\mathrm{CME}$, altered in response to experimentalpain?

Question 2. Is there a relationship between CME during experimental pain and pain severity?

\section{Search strategy}

Searches will be conducted in the following databases: Scopus, PubMed, MEDLINE, PsycINFO and Web of Science. Key words relating to experimental pain and TMS will be used. These terms will be used as either free or controlled terms to identify literature of interest and will be adjusted for each database. The full search strategy for each database can be viewed in Appendix 1. The reference lists of eligible studies will be searched to identify additional studies.

\section{Inclusion Criteria}


Studies will be included based on the following criteria:

- English Language

- Published in peer reviewed journal articles.

- Healthy adult human participants (age $>18$ years, with no gender restrictions).

- Experimentally induced cutaneous (skin) or musculoskeletal (muscle or joint) pain delivered to any area of the body. Examples of cutaneous pain paradigms include capsaicin heat, electrical stimulation, laser stimulation, cold water immersion or thermal heat models. Examples of musculoskeletal pain paradigms include nerve growth factor, delayed onset muscle soreness, absorbic acid and hypertonic saline models.

- Outcomes include assessment of one or moreof: $\mathrm{CME}$, intracortical, transcortical or sensorimotor circuits. These measures are described in detail in the primary outcomes section.

- Inclusion of a pre-pain baseline or pain-free control condition (in the same participants).

\section{Exclusion Criteria}

Studies will be excluded based on the following criteria:

- Experimental pain is induced in the presence of another intervention (e.g. during a motor or cognitive task) or active stimulation (e.g. repetitive TMS). Studies that use a shamstimulation condition during pain will be included as this is not expected to interact with corticomotor output.

- Grey literature including data from unpublished studies (e.g. graduate theses), published abstracts, book chapters and conference proceedings.

- Pain severity is not assessed, or it is not clear whether participants experienced pain.

- The timing of TMS outcomes relative to pain induction is not stated.

\section{Primary Outcomes}

Eligible studies should reportone of the following measures:

1) Measures of CME including the amplitude or area of MEPs elicited when TMS is delivered to the "hotspot" (the scalp location that generates the largest MEPs) or map volume of the corticomotorrepresentation of amuscle[5, 6, 13].

2) Measures of intracortical circuitry within $\mathrm{M} 1$, including short-interval intracortical inhibition (SICl), intracortical facilitation (ICF) and long-interval intracortical inhibition (LICI). These are assessed using paired-pulse TMS, where a conditioning pulse to M1 is delivered prior to a test stimulus to M1. Depending on the interval between stimuli, this causesinhibition or facilitationof CME relative to the test stimulus alone. SICloccurs at an interstimulus interval (ISI) of $1-6 \mathrm{~ms}$, ICF at an ISI of $10-30 \mathrm{~ms}$ andLICI at an ISI of 50-200ms[18, 19]. 
3) Measures of transcortical circuitry, including short-interval interhemispheric inhibition (SIHI) or longinterval interhemispheric inhibition (LIHI). These are assessed using dual-coil TMS, where a conditioning stimulus to M1 is delivered prior to a test stimulus to the opposite M1. This causes inhibition ofCME relative to the test stimulus alone. SIHloccurs at an ISI of $10 \mathrm{~ms}$ andLIHI at an ISI of $40 \mathrm{~ms}[20]$.

4) Measures of sensorimotor circuitry, including short afferent inhibition (SAI)and long afferent inhibition (LAI). These are assessed by delivering a conditioning electrical stimulus to a peripheral nerve prior to a test magnetic stimulus to M1. This causes inhibition of CME relative to the test stimulus alone. SAI occurs at an ISI of $20 \mathrm{~ms}$ and LAI at an ISI of 200ms [21, 22].

\section{Study Identification}

References will be managed using Endnote X9. Duplicate studies will be removed. The title and abstract of each study identified through the search will be evaluated against the inclusion and exclusion criteria. If there is uncertainty about the eligibility of a study, the full text of that article will be retrieved for further information. The full texts of the remaining articles will then be screened to determine study eligibility. Articles that are excluded during full text screening and the reasons for their exclusion will be recorded. Study identification will be conducted by two independent reviewers. Any disagreements will be resolved by consensus, and if unresolved, a third reviewer be consulted.

\section{Data Extraction}

Information such as sex, age, and main findings/outcomes (described in the data synthesis section) will be extracted. In addition, specific characteristics of the experimental pain paradigm will be extracted such as: pain model (transient, tonic or transitional) and painfultissue (cutaneous [23] or musculoskeletal[10]). TMS data to be extracted will include: stimulation intensities, interpulse intervals, target and non-target muscles, whether measures are taken with the muscle at rest or undergoing active contraction,muscle contraction level (if under active contraction) and relative timeto pain onset/resolutionthat TMS measures were assessed. A custom extraction form (Appendix 2), piloted by two independent reviewers on two studies not directly related to this review, will be used to extract data from the included studies. Any disagreements will be resolved through a third reviewer.

\section{Risk of Bias Assessment}

To assess risk of bias and methodological quality of the included studies, we will use a custom 7-item checklist (Appendix 3). Some of these items are drawn from the Downs and Black checklist[24], while others were custom-made to capture the quality of each study's pain methodology. We will also use another checklist[25] to assess the quality of the TMS methodology (Appendix 4). After piloting these checklists, two independent reviewers will use them to assess risk of bias, with any disagreement resolved by a third reviewer.

\section{Data Synthesis}


Group Level Data. For each type of pain model (e.g. transient, tonic or transitional), data will be grouped as musculoskeletal or cutaneous pain, and into data obtained from target and non-target/control areas. Data will then be organised into windows to capture commonly assessed time frames during and after recovery from pain (Figure 1). For example, in tonic pain models, MEPs have been measured 0-10[26], 1120[27] and 21-30[28] minutes after pain resolution.

To compute the effect size (Cohen's $d_{a v}$ ), the following equation will be used:

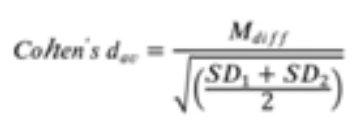

\section{(Equation 1)}

Where $M_{\text {diff }}$ refers to the mean difference between a pain/post-pain condition and a baseline/pre-pain condition, and $S D_{1}$ and $S D_{2}$ are the standard deviations for each of these conditions. $d_{a v}$ will be adjusted to Hedge's $g_{a w}$ using the following equation ( $n=$ sample size):

$$
\text { Hedges } g_{a v}=d_{a v} *\left(\frac{3}{4 * n-1}-1\right)
$$

\section{(Equation 2)}

Lastly, the variance in $g_{a v}\left(V g_{a v}\right)$ will be computed using the following equation ( $n_{1}$ and $n_{2}=$ sample size for the baseline and pain/post-pain condition):

$$
V g_{s v}=\left(\frac{n_{1}+n_{2}}{n_{1} n_{2}}\right)+\left(\frac{g_{a v}^{2}}{2\left(n_{1}+n_{2}\right)}\right)
$$

(Equation 3)

For within subjects designs, the correlation between dependent measures is usually required for effect size and variance calculations[29]. However, these values are usually not reported, in which case Equations 1-3,typically used for between subjects designs, can be used instead[30-32]. Where means and SDs of the outcome measures are not retrievable, an email will be sent to the corresponding author requesting these data. If there is no response, a decline to send data, or if authors are no longer contactable,means and SDs will be calculated from t-values, p-values and F-values, or estimated from illustrations.

Individual level data. For each study, the Pearson correlation coefficient ( $r$ )between CME change during pain (relative to baseline) and pain ratings will be determined. These data will be extracted from the study or obtained from raw data made available online (e.g., through an Open Science Framework). Where data are not openly available, an email will be sent to the corresponding author requesting this data.If there is no response, a decline to send data, or if authors are no longer contactable, then these studies will not be included in the individual level analysis. Individual level data will also be separated into pain model, 
painful tissue and target area.To estimate effect size from correlation coefficients, the value of $r$ for each study is not used. Instead, $r$ is converted to Fisher's z scale[29, 33] using the following equation:

$$
z=0.5 \times \ln \left(\frac{1+r}{1-r}\right)
$$

(Equation 4)

The variance in $z\left(V_{z}\right)$ is computed using the following equation [33]:

$$
V_{z}=\frac{1}{n-3}
$$

(Equation 5)

Meta-Analyses. The Meta-Analyses will be conducted using R and several packages (e.g., metafor, meta) [33]using the effect size $\left(g_{a v}\right.$ or $\left.z\right)$ and effect size variance values ( $V g_{a}$ or $\left.V_{z}\right)$ obtained from Equations 15 . Each effect size will be assigned a weight based on its variance, and the weighted $k$ effect sizes will be summed and then divided by the weights of the $k$ studies to calculate weighted mean effect size. The $95 \%$ confidence intervals of each weighted mean effect size will also be obtained. A z-transformation will be conducted to test the null hypothesis that the weighted mean effect size is 0 , with a two-tailed significance set at .05. The $Q$ statistic, to test whether there is significant heterogeneity amongst effect sizes, will be assessed against a $k-1$ Chi Squared distribution, with a $Q<.05$ indicating that the dispersion of effect sizes is substantial. The $R^{2}$ statistic will also be computed to estimate the percentage of variability due to effect size heterogeneity[34]. Substantial heterogeneity will be considered existent when $R>50 \%$. A minimum of 2 studies will be required for a meta-analysis. If there is only one study for an analysis, a narrative description of the findings will be provided.

Sensitivity Analysis. For each meta-analysis, a sensitivity analysis will be conducted by removing studies with high-risk of bias and re-running the meta-analysis. Issues related to sensitivity analyses are usually identified during the systematic review process[35]. For this reason, the exact cut-off value for what constitutes a high-risk study will be determined after piloting of the methodological checklists.

\section{Discussion}

This review will aid our understanding of mechanisms within the primary motor cortex that mediate pain. It will build on existing meta-analyses by determining whether alterations in CME in response to pain are influenced by different experimental pain paradigms. It will also assess the influence of experimental pain on intracortical, transcortical and sensorimotor modulators of CMEin response to experimental pain. Moreover, this review will provide the first exploration of individual differences in M1 activity in response to experimental pain. Understanding individual level data may be important for explaining why some people develop more severe pain than others following injury or illness.

\section{List Of Abbreviations}


M1: Primary Motor Cortex

TMS: Transcranial Magnetic Stimulation

MEP: Motor-Evoked Potential

CME: Corticomotor excitability

SICl: Short-Interval Intracortical Inhibition

LICl: Long-Interval Intracortical Inhibition

ICF: Intracortical Facilitation

SIHI: Short-Interval Interhemispheric Inhibition

LIHI: Long-Interval Interhemispheric Inhibition

SAl: Short-Afferent Inhibition

LAl: Long-Afferent Inhibition

\section{Declarations}

\section{Ethics Approval and Consent to Participate:}

Not Applicable

\section{Consent for Publication:}

Not Applicable

\section{Availability of Data and Materials:}

Not Applicable

\section{Competing Interests:}

The authors declare they have no competing interests

\section{Funding:}


SMS receives salary support from the National Health and Medical Research Council of Australia (1105040)

\section{Author's contributions:}

NSC drafted and edited the manuscript, search strategy, data extraction form and methodology checklist. W-JC edited the manuscript, search strategy, data extraction form and methodology checklist. SKM, PS and SMS edited the manuscript. KB and DSS made conceptual contributions.

\section{Acknowledgements:}

None

\section{References}

1. Vos, T., et al., Years lived with disability (YLDs) for 1160 sequelae of 289 diseases and injuries 19902010: a systematic analysis for the Global Burden of Disease Study 2010. The Lancet, 2012. 380(9859): p. 2163-2196.

2. Mercier, C., et al., Effect of Experimental Cutaneous Hand Pain on Corticospinal Excitability and Short Afferent Inhibition. Brain Sci, 2016. 6(4).

3. Li, L., et al., Changes of gamma-band oscillatory activity to tonic muscle pain. Neurosci Lett, 2016. 627: p. 126-31.

4. Schabrun, S.M., et al., Motor Cortex Reorganization and Impaired Function in the Transition to Sustained Muscle Pain. Cereb Cortex, 2016. 26(5): p. 1878-90.

5. Terao, Y. and Y. Ugawa, Basic Mechanisms of TMS. Journal of Clinical Neurophysiology, 2002. 19(4): p. 322-343.

6. Uy, J., M.C. Ridding, and T.S. Miles, Stability of maps of human motor cortex made with transcranial magnetic stimulation. Brain Topogr, 2002. 14(4): p. 293-7.

7. Delahunty, E.T., L.M. Bisset, and J.J. Kavanagh, Intracortical motor networks are affected in both the contralateral and ipsilateral hemisphere during single limb cold water immersion. Exp Physiol, 2019. 104(8): p. 1296-1305.

8. Alhassani, G., M.B. Liston, and S.M. Schabrun, Interhemispheric Inhibition Is Reduced in Response to Acute Muscle Pain: A Cross-Sectional Study Using Transcranial Magnetic Stimulation. J Pain, 2019. 20(9): p. 1091-1099.

9. Burns, E., L.S. Chipchase, and S.M. Schabrun, Reduced Short- and Long-Latency Afferent Inhibition Following Acute Muscle Pain: A Potential Role in the Recovery of Motor Output. Pain Med, 2016. 17(7): p. 1343-1352. 
10. Schabrun, S.M. and P.W. Hodges, Muscle pain differentially modulates short interval intracortical inhibition and intracortical facilitation in primary motor cortex. J Pain, 2012. 13(2): p. 187-94.

11. Summers, S.J., et al., Motor adaptation varies between individuals in the transition to sustained pain. Pain, 2019. 160(9): p. 2115-2125.

12. Costa, Y.M., et al., Masseter corticomotor excitability is decreased after intramuscular administration of nerve growth factor. Eur J Pain, 2019. 23(9): p. 1619-1630.

13. Seminowicz, D.A., T. Thapa, and S.M. Schabrun, Corticomotor Depression is Associated With Higher Pain Severity in the Transition to Sustained Pain: A Longitudinal Exploratory Study of Individual Differences. J Pain, 2019. 20(12): p. 1498-1506.

14. Burns, E., L.S. Chipchase, and S.M. Schabrun, Primary sensory and motor cortex function in response to acute muscle pain: A systematic review and meta-analysis. Eur J Pain, 2016. 20(8): p. 1203-13.

15. Chang, W.J., et al., Altered Primary Motor Cortex Structure, Organization, and Function in Chronic Pain: A Systematic Review and Meta-Analysis. J Pain, 2018. 19(4): p. 341-359.

16. Parker, R.S., et al., Is Motor Cortical Excitability Altered in People with Chronic Pain? A Systematic Review and Meta-Analysis. Brain Stimul, 2016. 9(4): p. 488-500.

17. Moher, D., et al., Preferred reporting items for systematic reviews and meta-analyses: the PRISMA statement. PLoS med, 2009. 6(7): p. e1000097.

18. Kujirai, T., et al., Corticocortical inhibition in human motor cortex. The Journal of Physiology, 1993. 471(1): p. 501-519.

19. Valls-Solé, J., et al., Human motor evoked responses to paired transcranial magnetic stimuli. Electroencephalography and Clinical Neurophysiology/Evoked Potentials Section, 1992. 85(6): p. 355-364.

20. Ferbert, A., et al., Interhemispheric inhibition of the human motor cortex. The Journal of Physiology, 1992. 453(1): p. 525-546.

21. Tokimura, H., et al., Short latency inhibition of human hand motor cortex by somatosensory input from the hand. The Journal of Physiology, 2000. 523(2): p. 503-513.

22. Chen, R., B. Corwell, and M. Hallett, Modulation of motor cortex excitability by median nerve and digit stimulation. Experimental Brain Research, 1999. 129(1): p. 77-86.

23. Fierro, B., et al., Repetitive transcranial magnetic stimulation (rTMS) of the dorsolateral prefrontal cortex (DLPFC) during capsaicin-induced pain: modulatory effects on motor cortex excitability. Exp Brain Res, 2010. 203(1): p. 31-8.

24. Downs, S.H. and N. Black, The feasibility of creating a checklist for the assessment of the methodological quality both of randomised and non-randomised studies of health care interventions. Journal of Epidemiology and Community Health, 1998. 52(6): p. 377-384.

25. Chipchase, L., et al., A checklist for assessing the methodological quality of studies using transcranial magnetic stimulation to study the motor system: An international consensus study. Clinical Neurophysiology, 2012. 123(9): p. 1698-1704. 
26. Rittig-Rasmussen, B., et al., The role of neuroplasticity in experimental neck pain: a study of potential mechanisms impeding clinical outcomes of training. 2014. 19(4): p. 288-293.

27. Le Pera, D., et al., Inhibition of motor system excitability at cortical and spinal level by tonic muscle pain. 2001. 112(9): p. 1633-1641.

28. Tsao, H., K.J. Tucker, and P.W. Hodges, Changes in excitability of corticomotor inputs to the trunk muscles during experimentally-induced acute low back pain. Neuroscience, 2011. 181: p. 127-33.

29. Borenstein, M., et al., Effect sizes based on means. Introduction to meta-analysis, 2009: p. 21-32.

30. Dunlap, W.P., et al., Meta-analysis of experiments with matched groups or repeated measures designs. Psychological methods, 1996. 1(2): p. 170.

31. Hirst, R.J., L. Cragg, and H.A. Allen, Vision dominates audition in adults but not children: A metaanalysis of the Colavita effect. Neuroscience \& Biobehavioral Reviews, 2018. 94: p. 286-301.

32. Lakens, D., Calculating and reporting effect sizes to facilitate cumulative science: a practical primer for t-tests and ANOVAs. Frontiers in psychology, 2013. 4: p. 863.

33. Harrer, M., et al., Doing meta-analysis in R: A hands-on guide. PROTECT Lab Erlangen, 2019.

34. Borenstein, M., et al., Effect sizes for continuous data. The handbook of research synthesis and meta-analysis, 2009. 2: p. 221-235.

35. Deeks, J.J., et al., Cochrane handbook for systematic reviews of interventions version 5.1. 0 (updated March 2011). The Cochrane Collaboration, 2011: p. 2.

\section{Figures}

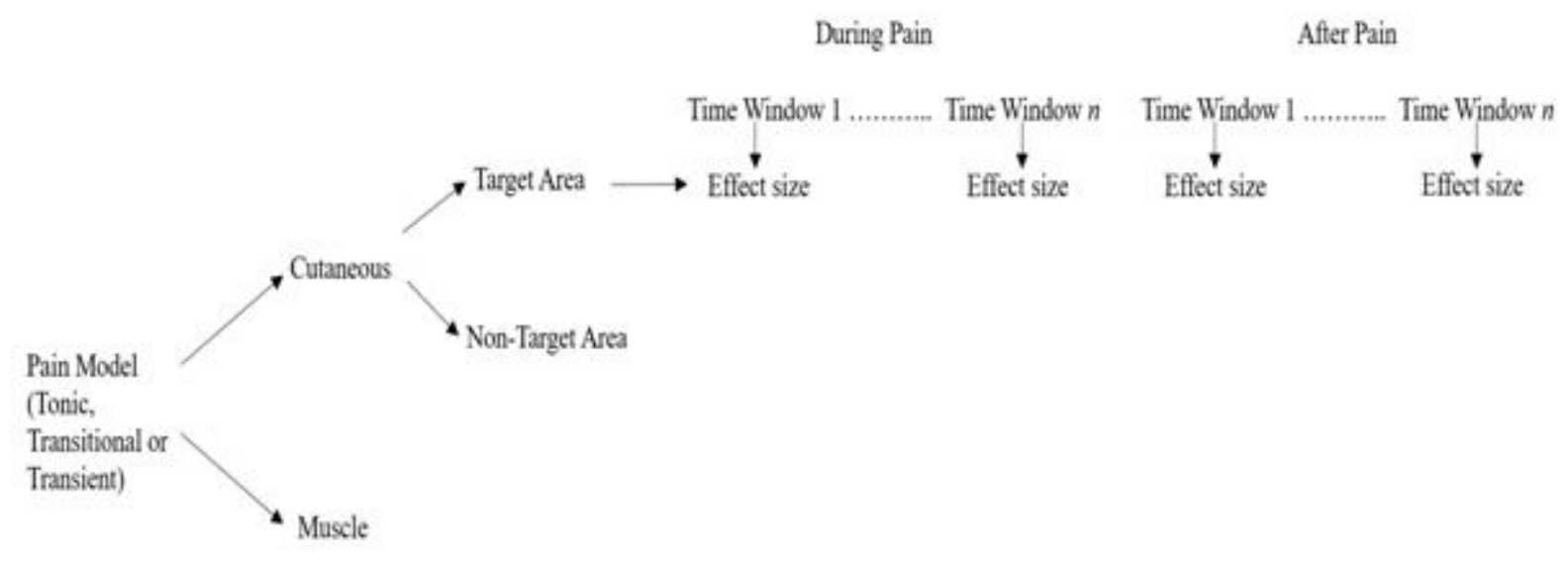

\section{Figure 1}

Diagrammatic representation of how effect sizes will be organised. An effect size will be computed for each time window (total number of time windows indicated by $\mathrm{n}$ ). Studies may use tonic, transitional or transient pain models, inflicting either cutaneous or musculoskeletal pain, while measuring MEPs from the target or non-target area. 


\section{Supplementary Files}

This is a list of supplementary files associated with this preprint. Click to download.

- Appendix1SearchTerms.docx

- Appendix3RiskofBiasChecklist.docx

- Appendix2ExtractionSheet.docx

- Appendix4TMSChecklist.docx 